\title{
Visit-to-visit blood pressure variability and cardiovascular and kidney diseases
}

\author{
Jerzy Głuszek', Teresa Kosicka² \\ 1State Higher Vocational School in Kalisz \\ 2Department of Arterial Hypertension, Vascular and Internal Diseases, University of Medical Sciences, Poznan
}

\begin{abstract}
In healthy people and patients with hypertension, blood pressure varies between clinic visits every several days, a week, or a month apart. Although long thought to be an artifact, recent data suggest that visit-to-visit variability of blood pressure is reproducible and has independent association with cardiovascular outcomes and mortality. There currently is no consensus on how to define blood pressure variability best. The most common metric used is standard deviation of mean blood pressure, variation independent of the mean and average real variability. Blood pressure variability is higher in female, older age and patients with hypertension, with cardiovascular diseases, with diabetes mellitus and chronic kidney disease. Therefore, we are not sure if higher blood pressure variability is the only marker of arterial stiffness and consequence of end organ damage or prognostic indicator of cardiovascular events. We also do not know whether reducing blood pressure variability would reduce the risk of cardiovascular outcomes. However, the number of studies on blood pressure variability is growing exponentially and perhaps in the near future, high blood pressure variability will also be the goal of hypertension therapy.

key words: blood pressure variability, cardiovascular diseases, kidney failure
\end{abstract}

Arterial Hypertens. 2016, vol. 20, no. 1, pages: 26-31

DOI: $10.5603 / A H .2016 .0006$

A great number of publications have long showed a significant association between high blood pressure and increased cardiovascular risk. The relationship between the variability in blood pressure and cardiovascular disease is much less known, and even objectionable. We can distinguish pressure variability in short periods of time (the "beat-to beat" variability), pressure variability between day and night and finally the pressure variability that occurs at longer intervals. The influence of absence of the physiological pressure drop in hours of sleep on the increase of risk of cardiovascular disease is well known [1]. Pressure variability in a very short periods of time is also the subject of a number of scientific reports and their discussion is beyond the scope of the current work. Variability in long periods of time can be detected through repeated every day, once a week, once a month or once every six months pressure measurements conducted by the doctor, the patient, or more often through repeated automatic measurement (ABPM). It has long been stated that blood pressure is usually slightly but significantly higher in winter and lower in summer [2]. Regardless of the influence of the outside temperature, a periodic variation in pressure between the pressure measurements over long periods of time is becoming a subject of rapidly growing number of publications. Initially it was thought that this variation is random and clinically not relevant. Munter $e t$ al. were the first to show that variability in long periods of time or variability between medical visits (visiting-to-visit variability) is repeatable [3]. Cited authors observed 772 patients with hypertension for almost 3 years and made 14 blood pressure measurements in each

Address for correspondence: Teresa Maria Kosicka, MD Department of Arterial Hypertension, Vascular and Internal Diseases, University of Medical Sciences, Poznan, Długa 1/2, 60-848 Poznań tel.: (061) 85490 90; fax: (061) 85490 90, e-mail: tkosicka@poczta.fm 
of them. Each of these patients had standard deviation of the first seven measurements of systolic blood pressure calculated, and the same calculations were made for the next seven measurements of systolic pressure and the existence of a statistically significant correlation between the standard deviation in both series of measurements was demonstrated. Patients qualified for the group of $20 \%$ of patients with the highest standard deviation in the first seven pressure measurements had a strong probability of recurring qualification for this group based on the subsequent seven measurements of blood pressure. Similar results were obtained by the cited authors using other measures of dispersion than the standard deviation. These data testify to the fact that assessed blood pressure variability is reproducible and it is not an accidental value. So far, no uniform guidelines to recommend the use of selected, most effective measures of variability have been developed, and now the authors of various publications on this subject use more than 10 different indicators evaluating the degree of variability [4]. The most commonly used measures of variability included: the standard deviation of the mean systolic or diastolic blood pressure, coefficient of variability, the maximum difference between the values of blood pressure (maximum blood pressure variability), variation independent of the mean [4]. Recently, more a frequently used indicator is referred to as "average real variability", as determined by the formula:

$$
\sum_{\mathrm{I}=1}^{\mathrm{N}-1}\left(\mathrm{X}_{\mathrm{i}+1}-\mathrm{X}_{\mathrm{i}}\right) \mathrm{I}(\mathrm{n}-1)
$$

where $\mathrm{Xi}$ is the level of blood pressure readings over multiple visits.

Some authors use the first derivative of variation in systolic blood pressure measured by ABPM, and show that the rate of pressure changes included in this method correlates in a better way with atherosclerotic changes in the organ [5].

In young patients, the variation in blood pressure in $50 \%$ of cases can be explained by genetic factors [6]; in the elderly, $80 \%$ variation in pressure depends on environmental factors [4]. Numerous studies have shown that variability of blood pressure is greater in old age [7], and in women than in men $[4,8]$. Conflicting results include the effects of obesity on blood pressure variation degree. Interesting, however, are the results of studies evaluating the size of variability of blood pressure in patients with cardiovascular disease.

Variability of blood pressure is greater in patients with hypertension than in healthy people. Mancia $e t$ al. studied a group of patients with hypertension in whom the 24-hour mean blood pressure was $159 \pm$ $5.7 \mathrm{~mm} \mathrm{Hg}$ and a group of people with normal pressure of an average of $112.9 \pm 2.1 \mathrm{~mm} \mathrm{Hg}$ [5]. They demonstrated that changes in blood pressure were faster in patients with hypertension and were $6.50 \pm$ $0.40 \mathrm{~mm} \mathrm{Hg} / \mathrm{s}$, and in healthy individuals they were $4.80 \pm 0.30 \mathrm{~mm} \mathrm{Hg} / \mathrm{s}(\mathrm{p}<0.05)$.

Zakopoulos et al. showed a significant correlation between the variability of blood pressure and the carotid intima-media thickness independent of mean pressure values and other confounders [9]. Central and Eastern Europe had BP-CARE examination analyzing blood pressure variability depending on the age of respondents, some coronary risk factors and experienced cardiovascular diseases [10]. In the study involving more than 6,000 patients with hypertension, the standard deviation and the coefficient of variation of the three blood pressure measurements were calculated. Patients with the highest coefficient of variation, as compared to patients with the lowest rate, were characterized by significantly higher levels of cholesterol and blood glucose levels, a higher incidence of resistant hypertension and more frequent history of cardiovascular disease [10]. Australian researchers calculated the variation of systolic blood pressure in 5,880 patients with hypertension based on an average of 8 measurements of pressure during 4 years [11]. Ten percent of patients with the highest degree of pressure variability with respect to $10 \%$ of the respondents with the lowest pressure variability were characterized by a 2.18 times greater risk of cardiovascular complications, 2.78 times greater risk of stroke, 4.11 times greater tendency to myocardial infarction and 4.79 times more frequent occurrence of failure of the heart. This increased risk remained statistically significant after taking into account numerous confounding factors such as gender, age of the respondents, the average blood pressure values and the way of treatment. The analysis of variability of blood pressure in 14,382 patients with renal failure was conducted by Gorostidi et al. [12]. The patients with only hypertension had the standard deviation of $13.6 \mathrm{~mm} \mathrm{Hg}$, in patients with hypertension and in the first stage of renal failure the value was 16.7 $\mathrm{mm} \mathrm{Hg}$ and in the fourth stage of renal failure it was $17.5 \mathrm{~mm} \mathrm{Hg}$, while in the fifth stage the value was $19.0 \mathrm{~mm} \mathrm{Hg}$ ( $\mathrm{p}<0.001$ for trend). The standard deviation for systolic blood pressure at night was even higher and ranged from $17.5 \mathrm{~mm} \mathrm{Hg}$ (with still normal renal function) to $23.8 \mathrm{~mm} \mathrm{Hg}$ during end-stage of renal disease. The relationship between blood pressure variability and the degree of renal failure was greater for night systolic and weaker for 
diastolic blood pressure. According to Tanner et al., the high-pressure variation can be partly explained by a higher average blood pressure in patients with renal failure than in healthy people [13].

The relationship between the incidence of stroke and blood pressure variation was demonstrated by Shimbo et al. [14]. These authors analyzed blood pressure variability in 58,228 women participating in the Women's Health Initiative study. Blood pressure values were measured every year for an average period of 5.4 years. At this time, the examined women experienced 997 strokes. The variability of systolic blood pressure (measured by standard deviation) was significantly higher (after taking into account confounding factors) in patients with stroke. This relationship was particularly pronounced in women with low blood pressure (below $120 \mathrm{~mm} \mathrm{Hg}$ ).

There is growing evidence that increased blood pressure variability is not only a marker of cardiovascular changes, but it is also a risk factor for future strokes or heart attacks. Already in 2010, Rothwell et al. showed high variability of blood pressure between consecutive medical visits, and strokes that followed [15]. They re-analyzed the results of a large study (ASCOT-BPLA) including patients with hypertension and an additional cohort of patients with a history of transient cerebral ischemia. In this group of patients from the ASCOT-BPLA study, treated for hypertension, high variability in systolic blood pressure was a significant risk factor for stroke or cardiac ischemia (HR 3.25, p < 0.0001). The cited authors also observed that tested relationships are better for variability of pressure measured in the traditional way, but also important for the ABPM measurements especially in younger people and lower values of mean blood pressure. In a cohort group of patients with transient cerebral ischemia, in those with the highest variability in systolic blood pressure (measured by value of standard deviation), another stroke occurred six times more frequently than in those with the lowest variability of pressure, and this relationship was independent of the average blood pressure $(\mathrm{p}<0,0001)$. An important risk factor also included the highest average arterial blood pressure $(\mathrm{p}<0.0001)$. Subsequent studies by other authors confirm the observations made by Rothwell et al. In 2015, a post hoc analysis of the results of the so far largest study of treatment of hypertension, namely ALLHAT, was conducted by Munter et al. [16]. They calculated the standard deviation of seven measurements of blood pressure in hypertensive patients without symptoms of cardiovascular disease, and then traced their fate to the end of the ALLHAT study. The patients experienced 606 strokes, 1,194 cardiac events, with 921 respondents that developed symptoms of heart failure, and 1,948 patients died. In a quarter of patients with the highest blood pressure variability in the standard deviation of systolic blood pressure was more than $14.4 \mathrm{~mm} \mathrm{Hg}$, in $1 / 4$ of patients with the lowest variation, the standard deviation was less than $6.5 \mathrm{~mm} \mathrm{Hg}$. The hazard ratio between the two groups for cardiac ischemic events was 1.3 (significant change), the overall mortality rate in the group with higher pressure variability was $56 \%$ higher, there was $46 \%$ increase in strokes, and finally $25 \%$ more patients were likely to develop heart failure (all statistically significant changes). Similar results were obtained by Vishram et al., analyzing the study of LIFE multicenter data [17]. They assessed the standard deviation and the range of variability of blood pressure measurements made at $6,12,18$ and 24 month of study in patients with hypertension and left ventricular hypertrophy participating in the LIFE study. In a multiple regression model, it was demonstrated that both the higher variability of systolic and diastolic blood pressure in the next 24 months of the study is a risk factor for stroke and a composite endpoint, which includes occurrence of death from cardiovascular causes, non-fatal myocardial infarction and stroke. In patients treated with losartan, the standard deviation of systolic blood pressure was slightly lower than in patients treated with atenolol $(10.2 \pm 6.0$ versus $10.9 \pm 6.2 \mathrm{~mm} \mathrm{Hg})$. Elevated concentration of natriuretic peptide in the serum frequently precedes myocardial damage. A large group of people (664), which is part of the overall Japanese population without prior heart disease, was studied by Satoh et al. [18]. The studied group had the concentration of natriuretic peptide defined and blood pressure variability determined with standard deviation. Increased blood pressure variability was predominant in patients with concentration greater than $125 \mathrm{pg} / \mathrm{ml}$ of peptide.

Blood pressure variability in long and very long periods of time was studied by Hastie et al., among 14,522 patients with hypertension [19]. The authors showed that increased blood pressure variability measured by "average real variability" is significantly associated with increased mortality from all causes and mortality associated with cardiovascular causes. In addition, the authors showed that the studied variability is higher in women, the elderly, and patients with chronic kidney disease.

Takao et al. observed a group of 629 patients with type II diabetes who were first hospitalized in 1995-1996 and then no symptoms of cardiovascular diseases were found in them [20]. By 2012, 66 of these patients had a heart attack or stroke. Increased 
blood pressure variability in the period prior to these events proved to be an important predictor of cardiovascular complications.

Interesting results were also obtained by Chang et al., who followed the incidence of cardiovascular events depending on the variability in blood pressure [21]. The study included 114,900 patients in the 3rd and 4th stages of renal failure (GFR between 15 and 59 $\mathrm{mL} / \mathrm{min}$ ) not on dialysis yet. Within 6 months, they determined the size of the variability in systolic blood pressure by a coefficient of variance, standard deviation and the average real variability. Over the next 12 months, 5,114 patients died and 582 had to start dialysis. In $1 / 5$ of patients with the highest blood pressure variability, deaths were by $22 \%$ significantly higher than in patients with the $1 / 5$ lowest variability of pressure. In contrast, $45 \%$ higher incidence of the need for dialysis in patients with high variability in blood pressure after taking into account many additional confounding factors turned out to be statistically insignificant. Hemorrhagic strokes occurred in the observed patients slightly more often than ischemic strokes and were almost twice as common (by $91 \%$ ) in patients with high variability of pressure, compared with those with the lowest blood pressure variability. The incidence of heart attacks and the emergence of heart failure in patients with kidney failure after taking into account numerous confounding factors proved to be much higher but statistically insignificant.

On the other hand, Japanese researchers observed a correlation between high blood pressure variability with subsequent renal failure. A population of almost 50, 000 people aged 40-74 years (mean age 61.7 years) for a period of three years was examined by a group of researchers led by Yano [22]. Exclusion criteria included patients with diabetes or renal disease (creatinine clearance less than $60 \mathrm{~mL} / \mathrm{min}$ or proteinuria). In all patients, blood pressure was measured periodically and its variability was determined using standard deviation and an "average real variability" of blood pressure. In subsequent periods, renal failure developed in $6.3 \%$ of patients. After taking into account many confounding factors such as mean arterial pressure, use of antihypertensive drugs and metabolic factors, high variability of blood pressure has proved to be an important risk factor for kidney failure. In a relatively small number of patients with peritoneal dialysis, Yokota et al. observed an inverse relationship between blood pressure variability and the amount of residual diuresis [23]. Pressure variability assessed on the basis of 12 consecutive pressure measurements and determined by the average real variability highly negatively and signifi- cantly statistically correlated with gradually decreasing amount of daily urine.

The impact of blood pressure variability on cardiovascular complications has become a subject of 2 meta-analyses. A meta-analysis of Stergiou et al. from 2014 , including 22 publications on the home pressure measurements, demonstrated that blood pressure variability based on these measurements is an important cardiovascular risk factor independent of mean pressure [24]. The authors point out, however, that no optimal frequency of pressure measurements in the patient's home has been developed so far, neither was determined the most useful indicator of pressure variability, and yet there is no threshold above which the pressure variability is excessive. In another meta-analysis, also from 2014, Diaz et al. [25] relied on 37 controlled studies that meet the following conditions: blood pressure variability calculated from at least 3 measurements of blood pressure in adults followed by a prospective observation regarding the occurrence of myocardial infarction, brain stroke, or death, and taking into account numerous confounding factors. The authors of that study observed that any increase in the standard deviation of a $5 \mathrm{~mm} \mathrm{Hg}$ was associated with a $27 \%$ increase in cardiac ischemic events, $17 \%$ increase of strokes and a $22 \%$ increase of total mortality. These changes are statistically significant but they are slight and their reliability is limited by the small number of analyzed publications. It should be emphasized that risk factor of cardiovascular events is only slightly stronger in non-dipping patients with hypertension than the risk factors associated with variability in pressure over longer periods of time [26].

On the other hand, Boggia et al. emphasize that although some measures of variability in blood pressure may indicate an increased cardiovascular risk and all-cause mortality, most important values include the average pressure and antihypertensive therapy [27].

The literature includes only a few publications that have attempted to explain the increased longterm blood pressure variability. In many patients with essential hypertension, the increased activity of the sympathetic nervous system is indicated. Coulson [28] decided to check the hypothesis that increased catecholamine excretion in the urine (due to increased activity of the sympathetic nervous system) correlates with increased blood pressure variability. In patients with essential hypertension, aged from 23 to 71 , he studied the variability of systolic and diastolic blood pressure measured by ABPM and excretion of normetadrenaline and metadrenaline. In this study, the author has demonstrated a statistically significant 
correlation between the variability in blood pressure measured by the value of standard deviation and ratio of the standard deviation to the average pressure, and the daily excretion of normetadrenaline $(r=0.493$, $\mathrm{p}<0.02$ ). It should be noted that the author did not observe a significant correlation between mean blood pressure and catecholamine excretion in the urine. In contrast, this relationship has been demonstrated between subjects' age and the blood pressure variability and the excretion of normetadrenaline.

According to Diaz et al., huge variability in blood pressure may increase the incidence of cardiovascular complications through adverse effects on the vascular endothelium [29]. The authors studied a group of 72 people with blood pressure lower than 160/ $/ 100 \mathrm{~mm} \mathrm{Hg}$, not smoking and with normal cholesterol, glucose and creatinine values, whose pressure variability was calculated based on three separate visits to doctors and based on ABPM study. All patients were subjected to ultrasound tests assessing the forearm brachial artery diameter in response to increased flow (FMD) and nitroglycerin administration. The authors showed a significant negative correlation between blood pressure variability and expansion of the brachial artery and a positive correlation after administration of nitroglycerin. Impaired endothelial function caused by increased blood pressure variability may, according to Diaz et al., be largely responsible for the increased number of cardiovascular complications. Significant endothelial dysfunction in patients with high long-term pressure variability was also observed by Chinese researchers [30]. A group of 174 patients with hypertension were subject to calculations of pressure variation within 12 months of periodic measurements and the ultrasound demonstrated an impaired expansion of the brachial artery (FMD study) in patients with increased pressure variability.

Long-term blood pressure variability is currently the subject of an increasing number of works that will probably soon assess more thoroughly its importance in the pathogenesis of complications of hypertension. There are also studies on such treatment of hypertension, which will help to reduce this variability. However, we should already be aware of the possible dangers that may be connected with excessive pressure variability in the long-term.

\section{References}

1. Kabat M. Hipertensjologia. Patogeneza, diagnostyka i leczenie nadciśnienia tętniczego. Ed. Więcek A., Januszewicz A., Szczepańska-Sadowska E., Prejbisz A. Medycyna Praktyczna 2015: 154.

2. Alperovitch A., Lacombe J-M., Hanon O. et al. Relationship between blood pressure and outdoor temperature in large sample of elderly individuals. Arch. Intern. Med. 2009; 169: 75-80.
3. Munter P., Joyce C., Levitan E.B. et al. Reproducibility of visit-to-visit variability of blood pressure measured as part of routine clinical care. J. Hypertens. 2011; 29: 2332-2338.

4. Hussein W.F., Chang T.I. Visit to visit variability of systolic blood pressure and cardiovascular disease. Curr. Hypertens. Rep. 2015; 17: 14 .

5. Mancia G., Paratri G., Castiglioni P. et al. Daily life blood pressure changes are steeper in hypertensive than in normotensive subjects. Hypertension 2003; 42: 277-282.

6. Yadav S. Genome-wide analysis of blood pressure variability and ischemic stroke. Stroke 2013; 44: 2703-2709.

7. Mancia G., Facchetti R., Parati G., Zanchetti A. et al. Visit-to-visit blood pressure variability, carotid atherosclerosis and cardiovascular events in the European Lacidipine Study on Atherosclerosis. Circulation 2012; 126: 569-578.

8. Muntner P. The relationship between visit-to-visit variability in systolic blood pressure and all-cause mortality in the general population: findings from NHANES III, 1988-1994. Hypertension 2011; 57: 160-166.

9. Zakopoulos N.A., Tsigoulis G., Barlas G. et al. Time rate of blood pressure variation is associated with increased common carotid artery intima-media thickness. Hypertension 2005; 45: 505-512.

10. Grassi G., Seravalle G., Maloberti A. et al. Within-visit BP variability in central and Eastern Europe: findings from the BP-CARE study. J. Hypertens. 2015; 33: 2250-2256.

11. Chowdhury E.K., Owen A., Krum H., Wing L.M., Nelson M.R., Reid C.M. Systolic blood pressure variability is an important predictor of cardiovascular outcomes in elderly hypertensive patients. J. Hypertens. 2014; 32: 525-533.

12. Gorostidi M., Sarafidis P., Sierra A.L. Blood pressure variability increases with advancing chronic kidney diseases stage. A cross-sectional analysis of 14,382 hypertensive patients from Spain. J. Hypertens. 2015; 33 (Suppl. 1): e40. Doi. 1097/01.hjh 0000467455.93515.1e.

13. Tanner RF.M., Shimbo D., Dreisbach A.W., Carson A.P., Fox E.R., Munthner P. Asscociation between 24-hour pressure variability and chronic kidney disease: a cross-sectional analysis of African Americans participating in the Jackson heart study. BMC Nephrol. 2015; 16: 84.

14. Shimbo D., Newman J.D., Aragaki A.K. et al. Association between annual visit-to visit blood pressure variability and stroke in postmenopausal women: Data from the Women's Health Initiative. Hypertension 2012; 60: 625-630.

15. Rothwell P.M., Howard S.C., Dolan E. et al. Prognostic significance of visit-to visit variability, maximum systolic blood pressure, and episodic hypertension. Lancet 2010; 375: 895-905.

16. Munter P., Whittle JK., Lynch A.L. et al. Visit-to-visit variability of blood pressure and coronary heart disease, stroke, heart failure, and mortality: a Cohort study. Ann. Intern. Med. 2015; 163: 325-338.

17. Vishram J.K.K., Dahlof B., Devereux B. et al. Blood pressure variability predicts cardiovascular events independently of traditional cardiovascular risk factors and target organ damage: a LIFE substudy. J. Hypertens, 2015; 33: 2422-2430.

18. Satoh M., Hosaka M., Asayama K. et al. Association between N-terminal pro B-type naturetic peptide and day-to-day blood pressure and heart rate variability in general population: the Ohasama study. J. Hypertens. 2015; 33: 1536-1541.

19. Hastie C.E., Jeemon P., Coleman H. et al. Long-term and ultra- long-term blood pressure variability during follow-up and mortality in 13522 patients with hypertension. Hypertension 201; 62: 698-705.

20. Takao T., Kimura K., Suka M. et al. Relationships between the risk of cardiovascular disease in type 2 diabetes patients and both visit-to-visit variability and time-to-effect differences in blood pressure. J. Diabetes Complications 2015; 29: 699-706.

21. Chang TG.I., Tabada G.H.,Yang J., Tan T.C., Go A.S. Visit-to-visit variability of blood pressure and death, end-stage renal disease, and cardiovascular events in patients with chronic kidney disease. J. Hypertens. 2016; 34: 244-252.

22. Yano Y., Fujtimoto S., Kramer H. et al. Long-term blood pressure variability, new-onset diabetes mellitus, and new-onset chronic kidney disease in the Japanese General population. Hypertension 2015; 66: 30-36. 
23. Yokota K., Sakurada T., Koitasbashi K., Shibagaki Y., Kario K., Kimura K. Association between residual kidney function and visit-to-visit blood pressure variability in peritoneal dialysis patients. Adv. Perit. Dial. 2015; 31: 49-53.

24. Stergiou G.S., Ntineri A., Kollias A., Ohkubo T., Imai Y., Parati G. Blood pressure variability assessed by home assessed: a systematic review. Hypertens. Res. 2014; 37: 565-572.

25. Taylor K.S., Heneghan C.J., Stevens R.J., Adams E.C., Nunan D., Ward A. Heterogeneity of prognostic studies of 24 hour blood pressure variability. PLOS ONE 2015; May 18 doi: 10.1371/journal. Pone 1236375.

26. Boggia J., Assayama K., Li Y., Hansen T.W., Mena L., Schutte R. Cardiovascular risk stratification and blood pressure variability on ambulatory and home blood pressure measurement Curr. Hypertens. Rep. 2014; 16: 470 doi: 10.1007/s11906-14-0470-8.

27. Coulson J.M. The relationship between blood pressure variability and catecholamine metabolites: a pilot study. J. Hum. Hypertens. 2015; 29: 50-52.

28. Diaz K.M., Veerabhadrappa P., Kashem M.A.,R.et al. Visit-to-visit and 24-h blood pressure variability: association with endothelial and smooth muscle function in African Americans. J Hum. Hypertens. 2013; 27: 671-677.

29. Liu Q Liu Y., Han J., Li J., Dong S. Impact of visit-to-visit blood pressure variability on vascular function in elderly hypertensive patients. Nan Fang Yi Ke Da Xue Xue Bao 2015; 35: $1320-1324$ 